\title{
A framework for national scenarios with varying emission reductions
}

\author{
Shinichiro Fujimori ${ }^{1,2,3 凶}$, Volker Krey $\mathbb{B}^{3}$, Detlef van Vuuren $\mathbb{C}^{4,5}$, Ken Oshiro ${ }^{1}$, \\ Masahiro Sugiyama $\mathbb{1}^{6}$, Puttipong Chunark7, Bundit Limmeechokchai ${ }^{7}$, Shivika Mittal ${ }^{8,9}$, \\ Osamu Nishiura', Chan Park ${ }^{10}{ }^{10}$, Salony Rajbhandari7, Diego Silva Herran ${ }^{2,11}$, Tran Thanh Tu ${ }^{12}$, \\ Shiya Zhao1, Yuki Ochi ${ }^{13}$, Priyardarshi R. Shukla9', Toshihiko Masui', Phuong V. H. Nguyen ${ }^{14}$, \\ Anique-Marie Cabardos ${ }^{3}$ and Keywan Riahi $\mathbb{1}^{3,15}$
}

\begin{abstract}
National-level climate actions will be vital in achieving global temperature goals in the coming decades. Near-term (20252030) plans are laid out in Nationally Determined Contributions; the next step is the submission of long-term strategies for 2050. At present, national scenarios underpinning long-term strategies are poorly coordinated and incompatible across countries, preventing assessment of individual nations' climate policies. Here we present a systematic and standardized, yet flexible, scenario framework varying 2050 emissions to build long-term national energy and climate mitigation scenarios. Applying the framework to six major Asian countries reveals individual challenges in energy system transformation and investment needs in comparable scenarios. This framework could be a starting point for comprehensive assessments as input to the Global Stocktake over the coming years.
\end{abstract}

T he Paris Agreement ${ }^{1}$ defines a long-term temperature goal for international climate policy: "holding the increase in the global average temperature to well below $2{ }^{\circ} \mathrm{C}$ above pre-industrial levels and pursuing efforts to limit the temperature increase to $1.5^{\circ} \mathrm{C}$ above pre-industrial levels". While this global goal defines the fundamental direction of international climate policy, its achievement critically depends on national actions and policy-making at the national level. As part of the Agreement, countries are required to submit Nationally Determined Contributions (NDCs) outlining their GHG emissions reduction efforts at the national scale. Within the Paris Agreement, there are several mechanisms to ensure that national actions align with the global goals; first, Parties are required to regularly report on their progress towards implementing their NDCs. These reports form part of the so-called Global Stocktake; that is, an assessment of the sum of the national contributions. Second, countries are also required to submit long-term strategies (LTSs) to the UNFCCC (also called mid-century strategies). Some countries have already submitted them, and others are preparing to do so (as of September 2020). Third, the Paris Agreement contains provisions to increase efforts over time, through what has been dubbed the ratchet mechanism. In summary, one objective of the Agreement is to have national actions aligned with long-term goals, with routine checks and revisions of short- to medium-term national goals and policies.
Model-based climate and emissions scenarios are pivotal instruments for determining whether proposed actions are in line with the long-term goals ${ }^{2,3}$. In the Fifth Assessment Report of the IPCC (AR5), over a thousand scenarios were summarized in the database, assessed and classified by both assumption and mitigation levels ${ }^{4}$. The Special Report on $1.5^{\circ} \mathrm{C}$ was also accompanied by a large set of global scenarios that depict emissions pathways through the twenty-first century ${ }^{5,6}$. Many global model scenarios have been developed under specific model intercomparison projects (MIPs) by sharing scenario implementation protocols that prescribe the characteristics of the scenarios (for example carbon budgets, technological availability). This allows systematic assessment of a set of research questions and robust insights into climate change mitigation $^{7-10}$.

In a similar way to the role of global emissions scenarios in international negotiations, national scenarios have widely contributed to national policy-making ${ }^{2}$. In several countries and regions this is done by national modelling teams, but results are largely disseminated in governmental reports or as internal information, and only occasionally shared in academic papers ${ }^{11-13}$. Taking Japan as an example: a task force was established to determine the 2020 emission target in 2008, and its recommendations were published in a book ${ }^{11}$ (only available in Japanese), while there was no official scenario assessment for the NDCs submission. MIPs exist not only at the national level (for the United States, China, Brazil and

Department of Environmental Engineering, Kyoto University, Kyoto, Japan. ${ }^{2}$ Center for Social and Environmental Systems Research, National Institute for Environmental Studies (NIES), Tsukuba, Japan. ${ }^{3}$ International Institute for Applied System Analysis (IIASA), Laxenburg, Austria. ${ }^{4}$ PBL Netherlands Environmental Assessment Agency, The Hague, the Netherlands. ${ }^{5}$ Copernicus Institute for Sustainable Development, Utrecht University, Utrecht, the Netherlands. ${ }^{6}$ Institute for Future Initiatives, The University of Tokyo, Tokyo, Japan. ${ }^{7}$ Sustainable Energy and Low Carbon Research Unit (SELC), Sirindhorn International Institute of Technology (SIIT), Thammasat University (TU), Pathum Thani, Thailand. ${ }^{8}$ Grantham Institute-Climate Change and the Environment, Imperial College London, London, UK. ${ }^{9}$ Global Center for Environment and Energy, Ahmedabad University, Ahmedabad, India. ${ }^{10}$ Department of Landscape Architecture, College of Urban Science, University of Seoul, Seoul, Korea. "Institute for Global Environmental Strategies (IGES), Hayama, Japan. ${ }^{12}$ Department of Environmental Engineering, International University-Vietnam National University Ho Chi Minh City, Ho Chi Minh City, Vietnam. ${ }^{13}$ E-Konzal, Osaka, Japan. ${ }^{14} \mathrm{Faculty}$ of Environment - Natural Resources and Climate Change, Ho Chi Minh City University of Food Industry, Ho Chi Minh City, Vietnam. ${ }^{15} \mathrm{Graz}$ University of Technology, Graz, Austria. ${ }^{凶}$-mail: fujimori.shinichiro.8a@kyoto-u.ac.jp 
Table 1 | Summary of the characteristics of global and national scenarios

\begin{tabular}{|c|c|c|}
\hline & Global scenarios & National scenarios \\
\hline Producers & IAMs & National energy/IAMs \\
\hline $\begin{array}{l}\text { Main users of the } \\
\text { research outcomes }\end{array}$ & $\begin{array}{l}\text { The IPCC, UNEP, UNFCCC, international and national } \\
\text { policymakers }\end{array}$ & $\begin{array}{l}\text { National policymakers, private companies, stakeholders and the } \\
\text { IPCC }\end{array}$ \\
\hline Main study target & $\begin{array}{l}\text { Global climate goals and the associated implications for } \\
\text { the climate, energy, the economy, land-use and so on }\end{array}$ & $\begin{array}{l}\text { Individual national climate goals/targets and their implications } \\
\text { for energy, the economy, land-use and so on }\end{array}$ \\
\hline Scenario implementation & $\begin{array}{l}\text { Individual studies or standardized modelling protocols } \\
\text { implemented by multiple models }\end{array}$ & Some standardization in projects, but mostly specific and varied \\
\hline Community organization & $\begin{array}{l}\text { Well established in the Integrated Assessment Modeling } \\
\text { Consortium (IAMC) }\end{array}$ & $\begin{array}{l}\text { Partially organized in different communities, often as part of a } \\
\text { modelling framework (such as The Energy Technology Systems } \\
\text { Analysis Program, but also to an extent in the IAMC) }\end{array}$ \\
\hline
\end{tabular}

$\operatorname{Japan}^{14-17}$ ), but also for specific regions such as the $\mathrm{EU}^{18}, \mathrm{Asia}^{19}$ and Latin America ${ }^{20}$. There have been a few attempts to collect national scenarios such as in the Linking Climate and Development PoliciesLeveraging International Networks and Knowledge Sharing project (CD-LINKS $^{21,22}$, which also includes individual national scenar$\left.\operatorname{ios}^{23-29}\right)$, the Climate Policy Assessment and Mitigation Modeling to Integrate National and Global Transition Pathways (COMMIT; https://themasites.pbl.nl/commit/) $)^{30}$ project and The Deep Decarbonization Pathways Project (DDPP) ${ }^{31,32}$. Moreover, various studies have assessed NDC implications, from sectoral perspectives $^{33-36}$ to the broader context of the UN Sustainable Development Goals ${ }^{37-40}$. It should also be noted that many countries do not have publicly available national energy or emissions scenarios.

Some major emitting countries rely on the scientific basis of existing national scenarios for national climate policy-making ${ }^{37,41}$, whereas many others do not. Furthermore, the emissions reduction targets of national scenarios are either determined by their own countries' interpretation of global goals (such as taking $2{ }^{\circ} \mathrm{C}$-consistent pathways and judging these by themselves) or are derived from global scenarios, such as those based on either cost-optimal scenarios or effort sharing schemes ${ }^{42}$ (left column in Table 1). Recent efforts made by national MIPs (for example, CD-LINKS) have shared a scenario protocol across countries based on global IAM results, but these allow only an assessment of specific conditions (for example, cost-optimal and global uniform carbon prices). Moreover, the modelling capability and the main strategies of GHG emissions reduction can be diverse across nations. Consequently, the level of emissions mitigation in national scenarios varies, which implies challenges for comparing mitigation costs and the degree of energy system changes across countries and scenarios. Apart from scenarios, real national emissions targets for both the near term and long term have often changed and will continue to do so in the future under various political and social circumstances. If only scenarios under specific, but limited, emissions reduction targets are available, the national scenarios quickly become outdated and irrelevant (see 'Complexity in the assessment of national scenarios').

Given this current situation, what if there were a standardized scenario framework that covers a wide range of emissions targets under the same reduction targets that are shared and implemented by many countries? For example, suppose that there were publicly available scenarios to reduce national emissions by $80 \%$ or $100 \%$ (not cumulative emissions) in 2050 for dozens of countries. What would the benefits for national policy-making of such a scientific basis standardized scenario framework be? There are at least four key benefits. First, it would reveal the dynamics of each nation's energy, land-use and agricultural systems, as well as economic implications, if the selected countries were to reduce emissions by similar levels. For example, a Japanese energy model comparison study was conducted, which found that even under the $80 \%$ reduction target in 2050, Japan would still have relatively high industrial sector energy demands because of its large dependency on heavy industry and limited renewable energy sources due to the small area of the country in comparison with the EU and the United States ${ }^{15}$. This kind of assessment would become available on a broader scale. Second, transparent publication in the scientific literature of the scenarios, the simulation models and how the scenarios were generated would contribute to ensuring that the scientific basis and quality of models and scenarios are maintained, to some extent, although this happens more frequently than before ${ }^{22}$. This is critical for evidence-informed policy-making. Third, this might allow a direct comparison of the challenges that countries are facing in achieving emissions reduction targets, which would be valuable when assessing the forthcoming national long-term climate targets from the perspective of social transition. Fourth, this would allow climate policymakers to compare each country's emissions targets and assess whether their own national targets are compatible with other countries' multiple reduction target possibilities or sufficient to reach the global long-term goals. Ultimately, policymakers may want to update the national targets; these are already supposed to undergo routine review as part of the Global Stocktake under the Paris Agreement. Although individual nations would have their own interests and priorities and the standardized simple scenario may not be sufficient to assess the national climate policies, such scenarios could at least be an entry point to communication with policymakers in many countries. From there, each country could build their own specialized and customized scenarios. We summarized the global and national modelling and scenarios circumstances in Table 1.

Here we present the issues with current national scenarios, propose a systematic and standardized scenario framework and demonstrate the implementation of such a framework for a few selected countries. Our proposal could ultimately contribute to the establishment of a central national scenario datahub for further national scenario assessments, similar to what has already been done for global scenarios (Table 1). We then discuss the complexity and expected criteria of national scenarios.

\section{Complexity in the assessment of national scenarios}

For short- to medium-term perspectives focusing on the next ten years, national policies and policy options, as well as stakeholder interests, are the primary concerns. In contrast, from a long-term perspective, a simple, comparable and systematic approach has clear benefits, facilitating a reassessment of the option space. It should be recognized that there are many determinants that are relevant for the specification of national emissions pathways, such as (1) global climate targets in the context of international commitments; (2) how to select global pathways in line with global long-term goals 
(for example, multi-IAMs uncertainty and physical climate science uncertainty); (3) selection of effort sharing schemes; (4) economic development stages in individual countries; and (5) other societal and development priorities that may be critical factors in determining the challenges of emissions reductions. The emissions reduction levels and challenges to achieving them naturally vary across countries and scenarios, and there is no need to have identical reduction levels across countries. The current NDCs, which are based on each nation's voluntary actions, are in many cases ambiguous, leading to significant uncertainty regarding the actual level of emission reduction targets ${ }^{43}$. This may or may not be because nations would prefer to keep some flexibility in the interpretation of their target statements, resulting in a degree of flexibility significant enough to change long-term global implications ${ }^{43}$. Either way, this would imply that, in principle, it is inevitable to have some degree of uncertainty in the actual national targets, and we should eventually develop strategies to cope with such uncertainties (more explanation for each source of uncertainty is given in Supplementary Note 1).

\section{Expected criteria for upcoming national scenarios}

Given the above-mentioned uncertainties, here we discuss the expected characteristics of the national scenarios, as listed below:

- Cross-national comparability

- Compatibility and cohesion with global climate goals

- Policy relevance

- Ability to address critical national target uncertainties

- Simple implementation without ambiguities in the interpretation of the modelling protocol

The comparability, which enables exploration of the relative stringency of national targets, is particularly beneficial for assessing national scenarios. One possible way to achieve this is fixed reduction rates across countries (for example, $80 \%$ reduction compared with a base year). The implications for the energy, land-use and economic transitions can reveal the associated challenges. Regarding the cohesion with global emissions pathways, global emissions scenarios with the climate goal specifications (for example, $2^{\circ} \mathrm{C}$ and $1.5^{\circ} \mathrm{C}$ ) in conjunction with effort sharing assumptions ${ }^{42}$ can bridge national and global scenarios. There can be considerable variation in national emissions pathways derived from the combination of effort sharing and global pathways, but we will show how our proposed framework can easily be mapped with global scenarios. For scenarios to be policy-relevant, the emission reduction levels should not be far from the targets laid out in forthcoming national LTSs. Exploring multiple mitigation levels has the advantage of identifying potential ambiguities in forthcoming LTSs, as well as enabling sensitivity analyses around the eventual LTS chosen. For example, supposing that the LTS for a country does not specify the GHG coverage but instead declares a 50\% reduction target in 2050, multiple scenarios would be mapped with full Kyoto gases $\left(\mathrm{CO}_{2}, \mathrm{CH}_{4}, \mathrm{~N}_{2} \mathrm{O}\right.$, hydrofluorocarbons (HFCs), perfluorocarbons (PFCs) and sulfur hexafluoride $\left.\left(\mathrm{SF}_{6}\right)\right)$ cases and $\mathrm{CO}_{2}$-only cases. A similar approach can be applied to other ambiguities. Finally, the simplicity of a modelling protocol that avoids ambiguities in its interpretation and the ease of implementing scenarios are of key importance to (1) allow such exercises to be performed in a decentralized manner, and (2) keep the barrier to joining such an effort as low as possible. The simplicity would facilitate updating of these scenario exercises on a regular basis, which will be discussed in the next section in more detail.

\section{Proposal for a systematic national scenario framework}

We thus propose a systematic and standardized approach for national scenarios that appropriately covers plausible future ranges of mitigation pathways and enables comparison across countries. Here we refer to this framework as national long-term pathways (NLPs), which comprises a set of national scenarios explained below. The role of NLPs could resemble Representative Concentration Pathways (RCPs) ${ }^{44}$ in formulating the ambition and range of climate targets. Following earlier works, we use the term 'scenario' to describe a plausible, comprehensive, integrated and consistent description of how the future might unfold, whereas the term 'pathway' is used for a set of scenarios ${ }^{45}$. This NLPs approach permits hedging against future national target uncertainties by not specifying a single emissions reduction target, but instead exploring multiple systematic scenarios associated with percentages of emissions reductions in 2050, the commonly considered target year for LTS, as a default set.

We classify two kinds of scenario. One is the so-called baseline, which excludes climate change mitigation policy but can include implemented and planned policies, as was done in earlier literature $^{2}$. Other socioeconomic assumptions are up to the individual modeller's choice, but we encourage avoidance of unusual specific assumptions such as without carbon capture and storage (CCS $)^{46}$ and low energy demands ${ }^{47}$. Although this modeller's choice might sometimes make the assessment and interpretation of the results difficult because socioeconomic backgrounds can differ among countries, there is an advantage in being able to skip a process to discuss what socioeconomic assumptions should be used and reach an agreement. More importantly, globally standardized socioeconomic scenarios such as the Shared Socioeconomic Pathways (SSPs) ${ }^{48}$ would not be most appropriate for individual countries, and thus the selection and assumption of socioeconomic conditions would depend on each country. If the national modellers cannot access national socioeconomic perspectives, the use of globally standardized SSPs would be recommended. The second kind of scenario is climate scenarios that target between $10 \%$ and $100 \%$ emissions reduction in 2050 compared with base year emissions, with $10 \%$ intervals covering the space between them. This can also be mapped with intensity targets, such as carbon intensity with gross domestic product (GDP) assumptions. For 2030, NDC targets can be adopted, but these may have variations associated with conditional/unconditional targets. Considering the current political situation, in which many countries are announcing carbon neutrality targets for different years that are not always 2050, our proposed emissions pathways can be easily extrapolated linearly beyond 2050 and can be assessed from the timing of zero emissions and the required transition towards that goal. If a model is unable to obtain feasible solutions for specific scenarios because emissions reductions are too strict, this information would also be reported. Energy-related $\mathrm{CO}_{2}$ emissions are the default emissions coverage. As we will discuss later, although there can be multiple options in the coverage of species and sectors (for example, full GHGs, including land sector), we chose specific emissions as defined above for two main reasons. First, energy-related $\mathrm{CO}_{2}$ emissions are the major source of emissions in most countries at present. Second, national modelling concerned with climate change mitigation policy is, in many cases, initiated from energy modelling, and considering developing countries whose modelling capability is relatively low, limiting the scope of coverage would be effective to enhance participation. Incorporation of other $\mathrm{CO}_{2}$ and non- $\mathrm{CO}_{2}$ emissions is not limited, because they are critical elements that determine the total GHG emissions. It is also important to design a holistic human system from energy, land-use and economic perspectives. The reduction percentages are relative to the specific base year (for example, 2010) for which the national emissions inventory is available for most countries and can thus exclude unnecessary uncertainties in the current NDCs. In this way, the NLPs proposal meets the criteria stated above, with comparability across countries, compatibility and cohesion with global climate goals, policy relevance and a relatively simple implementation protocol, and a strategy to address uncertainties. 
There should be flexibility in this proposal regarding at least the following two points. First, there are several options for emissions gas coverage. Full Kyoto GHGs would yield the best coverage, but sectoral and gas coverages can vary. For the gas coverage, this could include only $\mathrm{CO}_{2}$ or three major GHGs $\left(\mathrm{CO}_{2}, \mathrm{CH}_{4}\right.$ and $\left.\mathrm{N}_{2} \mathrm{O}\right)$. The sectoral coverage would be either full-sector or energy-related emissions only. This coverage should be considered depending on the availability of the information, composition of gases (for example, Brazil could have a large portion of emissions from the land-use sector) and modelling capability for each country. For non- $\mathrm{CO}_{2}$ emissions, the global warming potential (GWP), which is the heat absorbed by GHGs in the atmosphere as a multiple of the heat that would be absorbed by the same mass of $\mathrm{CO}_{2}$, should be standardized and a GWP100 metric should be used, as applied by UNFCCC and IPCC as the default choice in their reporting. Second, the reduction levels can be changed depending on country. For example, baseline emissions would not be increased for developed countries, whereas most developing countries can have much higher emissions in the future than now and starting the reduction percentage from $0 \%$ could still be deemed ambitious. For developed countries, more granularity might be needed for the range of deep reductions, and thus $5 \%$ intervals between $70 \%$ and $100 \%$ could be also attractive. The base year can also be flexible if needed (further flexibility options are given in Supplementary Table 1).

We also propose to routinely and periodically run this systematic scenario framework. In the global IAM community, there are series of almost routine-basis MIPs (for example, the Energy Modeling Forum (EMF)), which now have a large influence on global climate policies. In contrast, national scenarios are not yet so well established and can derive much more benefit from a scenario generation routine. There are multiple options for the routine intervals, such as every five years, every IPCC assessment cycle or according to international political milestones (for example, every Global Stocktake). The pros and cons of these choices can be considered later, but here we emphasize the advantages of having a regular scenario exercise under a similar protocol. First, the research community would be able to routinely provide policy-relevant information, tracing the model development history and tracking how the scenarios have changed over the period. Second, these regular exercises would allow individual countries' researchers to anticipate the forthcoming exercises and prepare a plan for model development, as well as take advantage of funding opportunities. In particular, this would be useful for developing countries where the energy models/IAMs are not yet fully developed. Note that it might be challenging to maintain completely harmonized protocols over time as political circumstances change (for example, the NDC process and its updates). The need for the routine exercise can also be extended to the global integrated assessment modelling community; the climate modelling community has such an experimental design, namely the Diagnostic, Evaluation and Characterization of Klima (DECK), under the umbrella of the Coupled Model Intercomparison Project (CMIP) ${ }^{49}$.

Keeping the scenario protocol simple is important and would enable modellers to implement the scenarios at regular intervals. In the meantime, in theory, tens of scenario variations depending on socioeconomic, technological availability/cost and policy assumptions could be developed. For example, SSPs in the global modelling community allow us to explore the variation of future socioeconomic assumptions ${ }^{48}$. Concerning variations in technological availability and cost, there are well-known examples in the global study carried out under EMF27 and the Assessment of Climate Change Mitigation Pathways and Evaluation of the Robustness of Mitigation Cost Estimates (AMPERE) projects ${ }^{46,50}$ (for example, non-CCS scenarios). Furthermore, there are similar national or regional implementations $s^{51-53}$. These scenario variations can be added to the standard set as supplementary (extended) scenarios in a similar way to those proposed in the SSPs ${ }^{54}$.
Regarding the relationships with policymakers, there are at least three main roles. First, for those countries that have not yet developed national scenarios, NLPs can provide opportunities to generate national scenarios, which would create dialogue between modellers and policymakers. Second, regardless of the existence of the national scenarios, comparable multinational scenarios can provide meaningful insights for each national policymaker, because national climate policy cannot be independent of the international context. These two benefits are valid for both the short and long term. Third, although it would be valuable to continue routine-based standardized scenario-making, more customization of the scenarios for each country might be needed in terms of socioeconomic assumptions and some specific national interests in the long term (for example, no more nuclear power in Japan). NLPs would then become an entry point for shifting from the standardized and systematic approach to creating such individual and unique national scenarios. Eventually, NLPs would provide a platform to maintain the national scenario modelling community that can enhance a dialogue among modellers and policymakers in a similar manner to CMIP.

\section{Demonstration of the proposal scenario design}

To explore how this newly proposed scenario set can be used, we have implemented the framework in selected Asian countries that have a large diversity in economic development stages, economy size and energy consumption patterns: China, India, Japan, Korea, Thailand and Vietnam. Each country individually runs national models, which means that countries do not change international market conditions. For scenario quantifications, we used the Asia-Pacific Integrated Model (AIM), which has been extensively applied in global and national climate change mitigation studies (Methods).

We first focus on an assessment of a single country: in this case, Japan (Fig. 1). The emissions in the baseline scenario are almost unchanged throughout the period, whereas climate mitigation scenarios, named CM30 to CM100, meet the NDC emission reduction target of $26 \%$ in 2030 and hit incremental $10 \%$ reductions levels as prescribed in the protocol in 2050 (Fig. 1a). Then, we compare projected emissions in 2050 with the global emissions pathways in conjunction with effort sharing schemes (Fig. 1b,c; Methods). As we consider the multiscenario uncertainties of global IAM emissions pathways for $1.5^{\circ} \mathrm{C}$ and $2{ }^{\circ} \mathrm{C}$ climate stabilization, at the national level, there is a large range of emissions levels associated with various effort sharing schemes (Fig. 1c). Here we also illustrate emissions target space with the long-term national goal for 2050, which in the case of Japan is an $80 \%$ reduction, but there is a range because the reference year and the GHG coverage are unspecified.

Then the energy system and economic implications for each emission reduction level are presented, which depend on the emissions reduction target levels (Fig. 1d-f). For example, the total energy supply is almost constant under a 30-60\% reduction, while the scenario with a $100 \%$ reduction in emissions implies a drop in supply by around half of the baseline. In other words, beyond $60-70 \%$ of emission reductions, a significant contribution of demand-side measures, including both energy efficiency improvements and behavioural change, are needed. Regarding the composition of energy sources, the contribution from low-carbon energy technology sources such as CCS and renewable energy sources gradually increase as reduction levels rise. The macroeconomic costs of mitigation increase considerably with more ambitious targets (Fig. 1e) and could rise to $3 \%, 4 \%$ and $4.5 \%$ of GDP losses with emission reduction targets of 80,90 and $100 \%$ in 2050 , respectively. Carbon prices are much more sensitive to reduction levels, increasing sharply to over US\$5,000 $\mathrm{tCO}_{2}^{-1}$ in a $100 \%$ reduction scenario, and to around US $\$ 2,000 \mathrm{tCO}_{2}^{-1}$ and $\mathrm{US} \$ 1,000 \mathrm{tCO}_{2}^{-1}$ in 90 and $80 \%$ reduction scenarios, respectively. The carbon price would become extremely high under stringent reduction targets, 
b

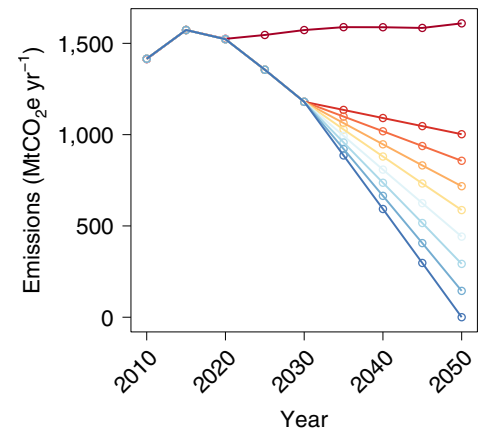

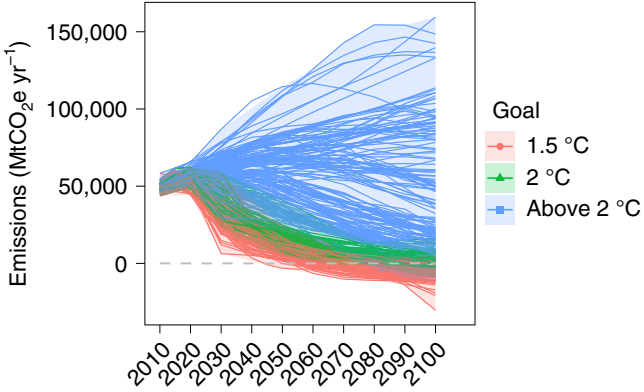

c

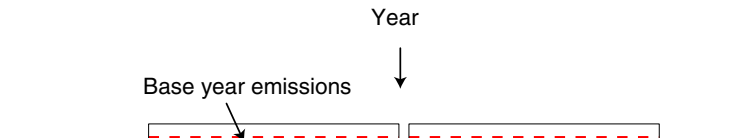

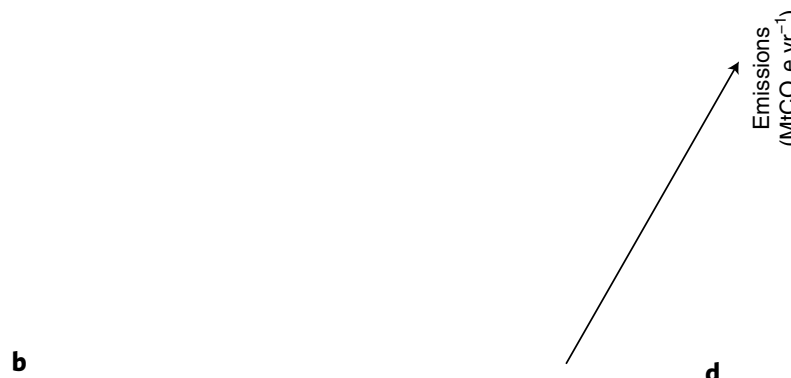

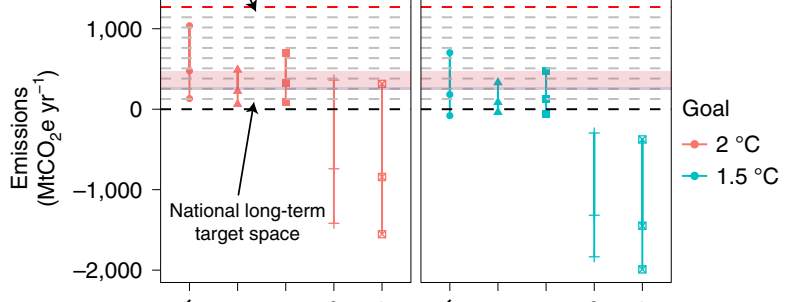

d

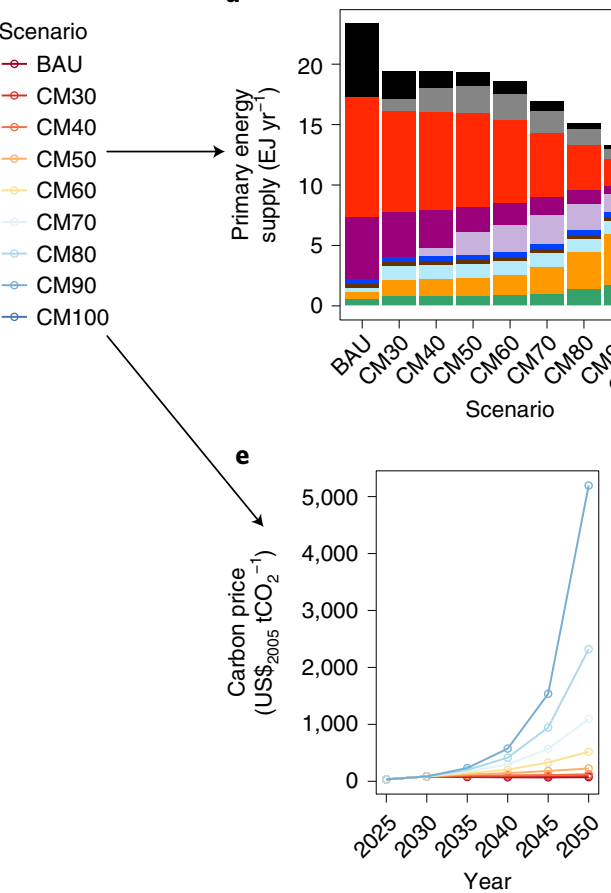

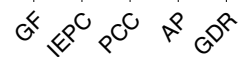

Approach

Approach
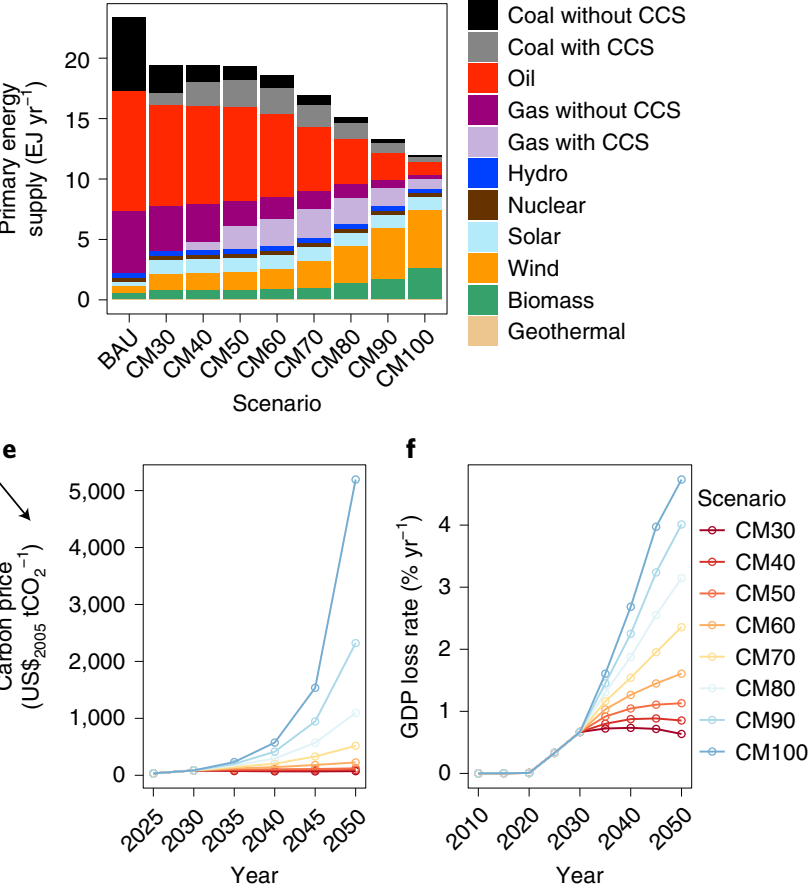

Fig. 1 | Illustrative example of the interpretation of the NLPs using Japan as a case study. a, Global emissions pathways under the scenarios indicated. b, National emissions pathways. c, Emissions in 2050 with a goal of $2{ }^{\circ} \mathrm{C}$ (left) or $1.5^{\circ} \mathrm{C}$ (right) of warming considering global pathways (all available scenarios are considered), effort sharing schemes and the long-term national goal of an $80 \%$ reduction. The uncertainty range (red shaded area) is associated with an unspecified reference year and gas coverages. The dashed red lines are emissions in 2010, and grey dashed lines correspond to incremental $10 \%$ reduction levels from base year emissions. d, Energy system implications represented by the primary energy supply and its composition in 2050. e,f, Policy cost implications in terms of carbon prices (e) and GDP loss rates (in terms of US $\$ 2005 ;$ ). AP, ability to pay; BAU, business-as-usual baseline scenario, ECPC, equal cumulative per capita emissions; GDR, greenhouse development rights; GF, grandfathering, IEPC, immediate per capita convergence, PCC, per capita convergence.

but this is due to the availability of negative emissions in Japan, where only a small area is left for energy crops and bioenergy combined with CCS. Below target reductions of $60 \%$, prices are lower than US $\$ 200 \mathrm{tCO}_{2}^{-1}$ over the period. More indicators are presented in Supplementary Fig. 1 for Japan and Supplementary Figs. 2-6 for other countries; several basic trends in many variables can be 
observed. There are gradual changes, with carbon price reductions in most cases, but it should be noted that there are some variables and countries for which convergences are apparent. For example, carbon prices and GDP losses in India and Vietnam display a trend that is due to the availability of CCS, including bioenergy combined with CCS. Once CCS becomes widely available, the carbon price is reduced. Final energy consumption in China, India and Vietnam therefore decreases along with the increasing rates of carbon price reduction in the 2020s and 2030s, but then converges in the 2040s. These results are due to the enhancement of electrification under mitigation $^{55}$, which offsets energy efficiency improvements.

Applying the framework to a country that submitted an LTS, the scenario outcomes could provide policymakers and analysts with an independent sensitivity around the LTS, which allows judgement of whether the targets are plausible or feasible from energy and economic perspectives. In addition, putting the LTS into the context of different equity principles sheds some light on the fairness of the target. However, policymakers need to interpret the results of model estimates carefully because they include uncertainties. The socioeconomic conditions were prescribed as SSP2 in this case, but the implications would change substantially if other conditions were assumed. Population and GDP are such key socioeconomic drivers, but technological availability and national energy policies are also sources of uncertainty. For example, unavailability of CCS pushes the policy cost much higher ${ }^{46}$, whereas low energy demand substantially mitigates the cost ${ }^{47}$. Finally, periodic reviews and assessments of the LTS, and the forthcoming 2035 or 2040 emissions reduction targets, will provide opportunities to revise and update the goals.

Regarding comparative assessments of multiple countries, in Fig. 2 we show selected indicators: the mean annual rate of energy intensity change (Fig. 2a), carbon intensity change (Fig. 2b), share of low-carbon energy sources (Fig. 2c), electrification rates (which is the final energy consumption of electricity divided by the total final energy consumption; Fig. 2d), carbon prices (Fig. 2e) and GDP loss rates (Fig. 2f). These indicators were chosen because they are fundamentally critical variables for assessing climate change mitigation, and as the scale of the economies, energy consumption and emissions of the countries assessed in this demonstration vary substantially; indicators that take percentages or relative (rather than absolute) values are more suitable for this analysis. We also carried out a regression analysis to clarify the common characteristics and the extent to which the reduction target rates in 2050 would change each indicator with dummy parameters, as shown in the Methods; these results are summarized in Fig. 2 and Supplementary Table 2.

We see a strong correlation with emissions reduction rates for most indicators except the mean annual rate of energy intensity change. The mean annual rate of carbon intensity change indicates $0.025 \%$ improvements per incremental $1 \%$ of emissions reduction. In contrast, the response of the mean annual rate of energy intensity change to reduction levels varies across countries, and the regressed slope is statistically insignificant. Japan's behaviour, in which energy intensity rises when increasing mitigation ambitions, is normal, whereas some other countries such as India, China and Vietnam seem to respond inversely. This is due to the requirement for negative emissions associated with bioenergy combined with CCS. This result would imply that improvements in carbon intensity are a common and effective strategy to reduce $\mathrm{CO}_{2}$ emissions, whereas energy efficiency improvements do not always yield the expected reduction in emissions. The share of low-carbon energy sources also shows a clear correlation with emissions reduction levels, and a $0.56 \%$ increase is expected per $1 \%$ of incremental emissions reduction. Electrification is a well-known and critical strategy for decarbonizing energy systems, and the regressed slope for change in electrification rates is $0.36 \%$. Note that for Korea and Vietnam (Supplementary Figs. 4 and 6), the time series of electrification in some mitigation scenarios cross in 2030s. In the near term, with modest emissions constraints, the electricity generation cost increases, which lowers electricity consumption while gas consumption increases. In the long term, under tighter emissions constraints, electrification needs to be enhanced. Carbon prices vary substantially by country, while the slope of regression is statistically significant at US $\$ 12.50 \mathrm{tCO}_{2}^{-1}$. Finally, the GDP loss rates would increase by $0.055 \%$ per $1 \%$ of additional emissions reductions. GDP loss rates also show variation across countries; Vietnam shows relatively high GDP loss rates, over $10 \%$, whereas Japan presents small values, less than $5 \%$ even in a $100 \%$ emissions reduction scenario. This variation comes from socioeconomic conditions such as the share of energy and food expenditures, which is largely influenced by abatement of non- $\mathrm{CO}_{2}$ emissions from the agricultural sector and the imposition of a carbon tax on them (if these are large, the relative influence on industrial structures and household consumption patterns would be large) and GDP per capita (if low, the carbon price intervention effects would be large), as well as assumptions on the availability of technology. It could be argued that this regression analysis would be affected by extreme country data. To test this, we conducted a sensitivity analysis to determine the robustness by withdrawing one country from the regression and then iterating the results for all countries. The results indicated that the carbon price and some other indicators were affected by the Japanese data (Supplementary Fig. 7).

Note that this study uses single model results. The use of multiple models, including multiple types of model (for example, top-down and bottom-up, or computable general equilibrium (CGE) and energy system models) could lead to different results ${ }^{56}$, which would enrich the implications of the study by introducing diversity in future prospects, and, in particular, might not indicate the clear relationships shown here.

\section{Caveats to the proposal and discussion}

We recognize that there are potential limitations to our proposal. First, policy relevance is the primary concern for this approach. This scenario set with its incremental $10 \%$ reduction levels might not exactly match the forthcoming LTS. As discussed, even if one of the scenario values of reduction rates hits a target, there will still be uncertainty in the inventory of the base year and coverage of GHGs. Second, there need to be several model runs (around 10 or more). However, in contrast to existing large-scale global models, national models tend to have relatively small computational loads, which could allow a relatively large number of scenarios to be run. In this sense, it is crucial to maintain the simplicity of the scenario requirements, as the simple scenario protocol allows researchers to systematically deal with scenarios running in the programming codes. To manage these issues, we view this proposal as a default core standard set, to which supplementary scenarios can be added, such as using varying technological availability taking into account individual countries' circumstances ${ }^{46}$. Moreover, NDCs can be updated, and ambitious LTSs may motivate countries to achieve more reductions in the near term, which would pose the question of whether more variations should be added in near-term reduction targets. Although such scenarios are excluded from this study, the updated NDC scenario could also be another set of supplementary scenarios. It is worth noting that such additional scenarios would have different roles from the above-proposed scenario set, and would require additional work to check and maintain the quality of results. Third, the protocol ignores possible interactions with the rest of the world. Increasing ambitions in one country might go hand in hand with actions in other countries. This could lead to impacts across countries. For example, fossil fuel prices could be low if many major countries decarbonized their economies. International price scenarios derived from global IAMs could be used as boundary conditions for national models, and in such a case, global models should also provide multilevel mitigation scenarios that could be prescribed by 

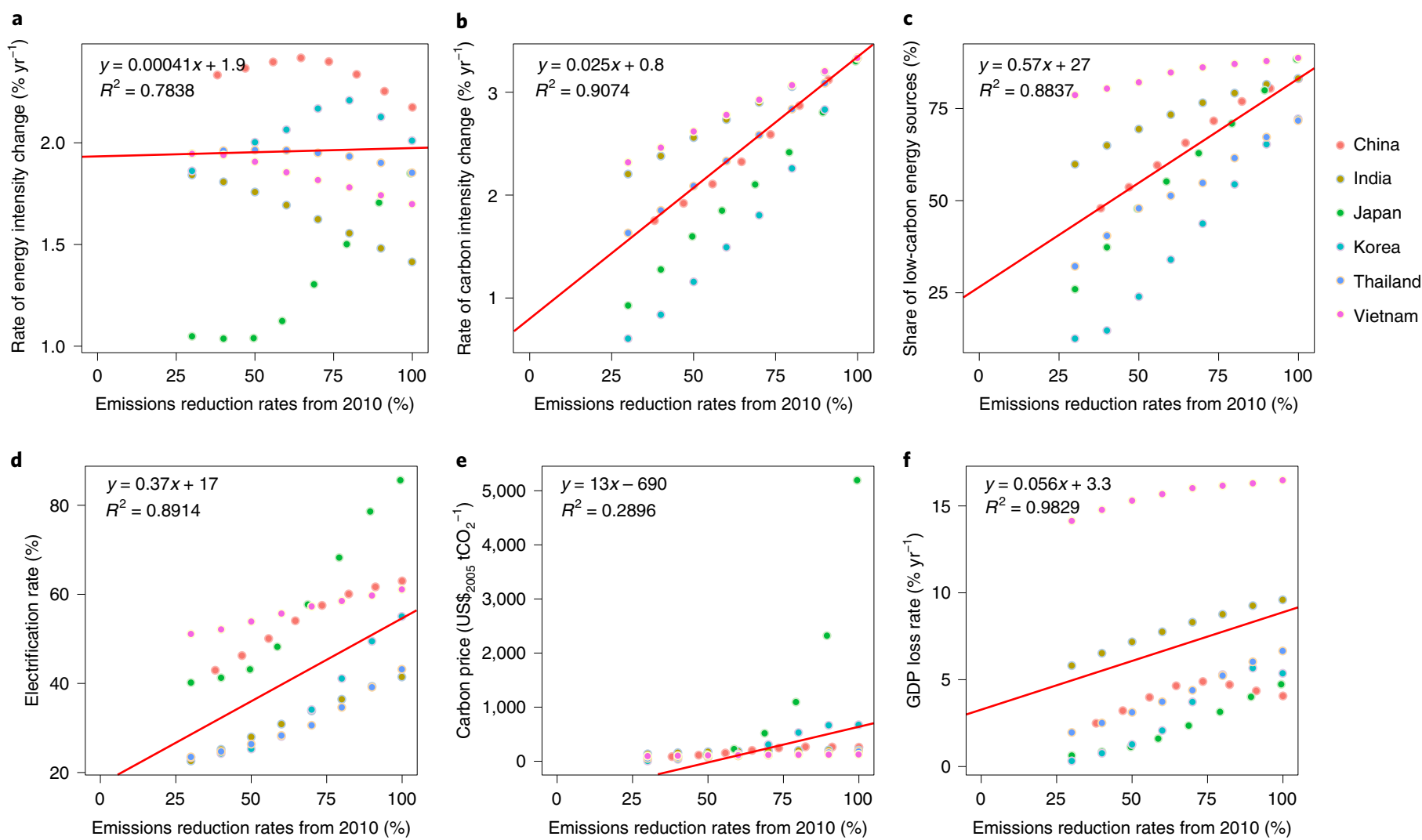

Fig. 2 | Cross-national comparison of NLPs. Six scenario indicators for 2050 are plotted against reduction targets. a, Mean annual rate of energy intensity change. b. Mean annual rate of carbon intensity change. c, Share of low-carbon energy sources in the primary energy supply. $\mathbf{d}$, Electrification rates in final energy consumption. e, Carbon prices. f, GDP loss rates. The solid lines indicate regression results using the derived slope, intercept and mean of dummy country results shown in Supplementary Table 2.

carbon budgets ${ }^{6}$. Still, the most direct impacts of more ambitious targets are nearly always felt simply within each country—and thus should serve as a caveat in the light of proposed simplification. Future study will be needed to investigate cross-border impacts. Fourth, the proposed scenarios always come with the risk of being outdated at some point, which can be critical in some cases. For example, long-term strategies were supposed to be submitted by the year 2020, and our proposal may not be able to keep up with them. Another possibility is that some extreme economic, social and political events may completely change the relevant energy-economic system. The disaster at the Fukushima nuclear power plant was one such turning point, and the COVID-19 pandemic has the potential to be another. A financial crisis, in general, could also result in structural change, which may imply that additional scenarios may be needed to take these extreme (or simply outlier) events into account. However, this depends on individual events and national circumstances. It may not be possible to generalize, and specific scenarios will probably need to be generated to address such events.

Finally, the current proposal could be a first step towards systematic national scenarios, much as global scenarios are stored and utilized effectively now. Meanwhile, even if the scenarios are developed by many countries, building up a valuable database, there would still be the need for better communication with policymakers. This is obvious from global IAM exercises. Even though there have been efforts to create transparent models ${ }^{57}$ and socioeconomic assumptions behind scenarios ${ }^{58}$, as well as making code open-source ${ }^{59,60}$ consistent with the recent demand for transparency, there is still an increasing demand to explain scenarios to decision-makers. Furthermore, the misinterpretation of current scenarios is an ongoing problem; for example, in the lack of climate change impacts ${ }^{61}$.
Therefore, just developing national scenarios is not sufficient, and better translation and communication of the scenarios to the policymakers is still needed.

\section{Community and capacity development}

The development of national scenarios fundamentally requires the involvement of researchers from each country. Many countries, including developing countries, have national models, but there are also many countries still lacking national energy models or IAMs. Even if national models exist, a certain portion of models need to improve their systematic model output reporting and model validation (including diagnostics and documentation), and will require considerable work to reach state-of-the-art modelling representation. In many cases, global integrated assessment modelling activities and experiences accumulated in the IAMC (https://www.iamconsortium.org/scientific-working-groups/ evaluation-and-diagnostics/) community should greatly help in the development of national modelling capacity ${ }^{57,60,62-65}$. Note that global models are themselves not always best; some national models have much more granularity in the representation of geographical and temporal resolutions, taking advantage of relatively smaller model coverage ${ }^{5,66}$. IAMC members have been actively involved in capacity development (for example, for Asian ${ }^{67}$ and Latin American ${ }^{16}$ capacity building activities, the National Institute for Environmental Studies Japan and Pacific Northwest National Laboratory have taken part in some exercises), and the IAMC itself sometimes coordinates these efforts. However, this proposed standardized scenario exercise could be a more meaningful and practical catalyst for enhancing capacity building activities within the climate mitigation modelling community. 


\section{Conclusions}

In this Perspective, we propose a new systematic and standardized scenario framework for long-term national scenarios and discuss its rationale, advantages and possible disadvantages. We believe that this proposal is valid and useful for policy-making and building a research community. National climate change mitigation modelling and scenario implementations might inherently have had relatively little motivation for building up a research community and conducting cross-national comparisons in the past. However, political and societal conditions have changed over the past decade, and we believe that national countermeasures are now a necessity for combatting climate change. The climate policy circumstances and need for national modelling and scenarios are expected to continue for at least the next couple of decades until emissions drop to sufficiently low levels. This research community should, therefore, devote much more attention and resources to national scenarios that guide or enhance actual transformative movement of societies. We envisage that the proposed framework could be a great milestone for national climate policy research and that many countries and models would engage with it. Thus, we call for community-level activities that will let a wide range of researchers involved in national climate policy assessment consider dedicating efforts to these important new activities.

\section{Online content}

Any methods, additional references, Nature Research reporting summaries, source data, extended data, supplementary information, acknowledgements, peer review information; details of author contributions and competing interests; and statements of data and code availability are available at https://doi.org/10.1038/ s41558-021-01048-z.

Received: 19 September 2020; Accepted: 13 April 2021; Published online: 27 May 2021

\section{References}

1. Adoption of the Paris Agreement. Proposal by the President (1/CP21) (UNFCCC, 2015); http://unfccc.int/resource/docs/2015/cop21/eng/ 10a01.pdf

2. Roelfsema, M. et al. Taking stock of national climate policies to evaluate implementation of the Paris Agreement. Nat. Commun. 11, 2096 (2020).

3. Emissions Gap Report 2018 (UNEP, 2019).

4. Clarke, L. et al. in Climate Change 2014: Mitigation of Climate Change (eds Edenhofer, O. et al.) 413-510 (IPCC, Cambridge Univ. Press, 2014).

5. Rogelj, J. et al. In IPCC Special Report on Global Warming of $1.5^{\circ} \mathrm{C}$ (eds Masson-Delmotte, V. et al.) 93-174 (WMO, 2018).

6. Fujimori, S., Rogelj, J., Krey, V. \& Riahi, K. A new generation of emissions scenarios should cover blind spots in the carbon budget space. Nat. Clim. Change 9, 798-800 (2019).

7. Bauer, N. et al. Global energy sector emission reductions and bioenergy use: overview of the bioenergy demand phase of the EMF-33 model comparison. Climatic Change 163, 1553-1568 (2018).

8. Luderer, G. et al. Residual fossil $\mathrm{CO}_{2}$ emissions in $1.5-2{ }^{\circ} \mathrm{C}$ pathways. Nat. Clim. Change 8, 626-633 (2018).

9. Fujimori, S. et al. A multi-model assessment of food security implications of climate change mitigation. Nat. Sustain. 2, 386-396 (2019).

10. McCollum, D. L. et al. Energy investment needs for fulfilling the Paris Agreement and achieving the Sustainable Development Goals. Nat. Energy 3, 589-599 (2018).

11. Fukui, T. Explanation of the Mid-Term Target for Global Warming Countermeasures (in Japanese) (Gyosei, 2009).

12. A Clean Planet for All-A European Long-Term Strategic Vision for a Prosperous, Modern, Competitive and Climate Neutral Economy. In-Depth Analysis in Support of the Commission Communication COM(2018) 773 (European Commission, 2018).

13. The White House United States Mid-Century Strategy for Deep Decarbonization (UNFCCC, 2016).

14. Fawcett, A. A., Clarke, L. E. \& Weyant, J. P. Introduction to EMF 24. Energy J. 35, 1-8 (2014).

15. Sugiyama, M. et al. Japan's long-term climate mitigation policy: multi-model assessment and sectoral challenges. Energy 167, 1120-1131 (2019).
16. Lugovoy, O. et al. Multi-model comparison of $\mathrm{CO}_{2}$ emissions peaking in China: lessons from CEMF01 study. Adv. Clim. Change Res. 9, 1-15 (2018).

17. Lucena, A. F. P. et al. Climate policy scenarios in Brazil: a multi-model comparison for energy. Energy Econ. 56, 564-574 (2016).

18. Weyant, J., Knopf, B., De Cian, E., Keppo, I. \& van Vuuren, D. P. Introduction to the EMF28 study on scenarios for transforming the European energy system. Clim. Change Econ. 04, 1302001 (2013).

19. Calvin, K. et al. The role of Asia in mitigating climate change: results from the Asia modeling exercise. Energy Econ. 34, S251-S260 (2012).

20. van der Zwaan, B. C. C., Calvin, K. V. \& Clarke, L. E. Climate mitigation in Latin America: implications for energy and land use: preface to the special section on the findings of the CLIMACAP-LAMP project. Energy Econ. 56, 495-498 (2016).

21. Schaeffer, R. et al. Comparing transformation pathways across major economies. Climatic Change 162, 1787-1803 (2020).

22. Schaeffer, R., Bosetti, V., Kriegler, E., Riahi, K. \& van Vuuren, D. Climatic change: CD-LINKS special issue on national low-carbon development pathways. Climatic Change 162, 1779-1785 (2020).

23. Köberle, A. C., Rochedo, P. R. R., Lucena, A. F. P., Szklo, A. \& Schaeffer, R. Brazil's emission trajectories in a well-below $2^{\circ} \mathrm{C}$ world: the role of disruptive technologies versus land-based mitigation in an already low-emission energy system. Climatic Change 162, 1823-1842 (2020).

24. Wang, H., Chen, W., Zhang, H. \& Li, N. Modeling of power sector decarbonization in China: comparisons of early and delayed mitigation towards 2-degree target. Climatic Change 162, 1843-1856 (2020).

25. Vishwanathan, S. S. \& Garg, A. Energy system transformation to meet NDC, $2{ }^{\circ} \mathrm{C}$, and well below $2{ }^{\circ} \mathrm{C}$ targets for India. Climatic Change 162, 1877-1891 (2020).

26. Mathur, R. \& Shekhar, S. India's energy sector choices-options and implications of ambitious mitigation efforts. Climatic Change 162, 1893-1911 (2020).

27. Feijoo, F., Iyer, G., Binsted, M. \& Edmonds, J. US energy system transitions under cumulative emissions budgets. Climatic Change 162, 1947-1963 (2020).

28. Safonov, G. et al. The low carbon development options for Russia. Climatic Change 162, 1929-1945 (2020).

29. Oshiro, K. et al. Mid-century emission pathways in Japan associated with the global $2^{\circ} \mathrm{C}$ goal: national and global models' assessments based on carbon budgets. Climatic Change 162, 1913-1927 (2019).

30. Fragkos, P. et al. Energy system transitions and low-carbon pathways in Australia, Brazil, Canada, China, EU-28, India, Indonesia, Japan, Republic of Korea, Russia and the United States. Energy 216, 119385 (2021).

31. Waisman, H. et al. A pathway design framework for national low greenhouse gas emission development strategies. Nat. Clim. Change 9, 261-268 (2019).

32. Bataille, C., Waisman, H., Colombier, M., Segafredo, L. \& Williams, J. The Deep Decarbonization Pathways Project (DDPP): insights and emerging issues. Clim. Policy 16, S1-S6 (2016).

33. Islas-Samperio, J. M., Manzini, F. \& Grande-Acosta, G. K. Toward a low-carbon transport sector in Mexico. Energies 13, 84 (2020).

34. Fyson, C. L. \& Jeffery, M. L. Ambiguity in the land use component of mitigation contributions toward the Paris Agreement goals. Earth Future 7, 873-891 (2019)

35. Maraseni, T. N. et al. Mapping national REDD+ initiatives in the Asia-Pacific region. J. Environ. Manage. 269, 110763 (2020).

36. Goes, G. V., Schmitz Gonçalves, D. N., de Almeida D’Agosto, M., de Mello Bandeira, R. A. \& Grottera, C. Transport-energy-environment modeling and investment requirements from Brazilian commitments. Renew. Energy 157, 303-311 (2020).

37. Iyer, G. et al. Measuring progress from nationally determined contributions to mid-century strategies. Nat. Clim. Change 7, 871-874 (2017).

38. Liu, J.-Y. et al. Identifying trade-offs and co-benefits of climate policies in China to align policies with SDGs and achieve the $2{ }^{\circ} \mathrm{C}$ goal. Environ. Res. Lett. 14, 124070 (2019).

39. Dal Maso, M., Olsen, K. H., Dong, Y., Pedersen, M. B. \& Hauschild, M. Z. Sustainable development impacts of nationally determined contributions: assessing the case of mini-grids in Kenya. Clim. Policy 20, 815-831 (2020).

40. Nogueira, L. P., Longa, F. D. \& van der Zwaan, B. A cross-sectoral integrated assessment of alternatives for climate mitigation in Madagascar. Clim. Policy 20, 1257-1273 (2020).

41. Weitzel, M. et al. Model-based assessments for long-term climate strategies. Nat. Clim. Change 9, 345-347 (2019).

42. van den Berg, N. J. et al. Implications of various effort-sharing approaches for national carbon budgets and emission pathways. Climatic Change 162, 1805-1822 (2019).

43. Rogelj, J. et al. Understanding the origin of Paris Agreement emission uncertainties. Nat. Commun. 8, 15748 (2017).

44. van Vuuren, D. P., Edmonds, J. A., Kainuma, M., Riahi, K. \& Weyant, J. A special issue on the RCPs. Climatic Change 109, 1-4 (2011).

45. van Vuuren, D. P. et al. A new scenario framework for climate change research: scenario matrix architecture. Climatic Change 122, 373-386 (2014). 
46. Kriegler, E. et al. The role of technology for achieving climate policy objectives: overview of the EMF 27 study on global technology and climate policy strategies. Climatic Change 123, 353-367 (2014).

47. Grubler, A. et al. A low energy demand scenario for meeting the $1.5^{\circ} \mathrm{C}$ target and sustainable development goals without negative emission technologies. Nat. Energy 3, 515-527 (2018)

48. Riahi, K. et al. Locked into Copenhagen pledges-implications of short-term emission targets for the cost and feasibility of long-term climate goals. Technol. Forecast. Soc. Change 90, 8-23 (2015).

49. Eyring, V. et al. ESMValTool (v1.0) - a community diagnostic and performance metrics tool for routine evaluation of Earth system models in CMIP. Geosci. Model Dev. 9, 1747-1802 (2016).

50. Riahi, K. et al. Locked into Copenhagen pledges-implications of short-term emission targets for the cost and feasibility of long-term climate goals. Technol. Forecast. Soc. Change 90, 8-23 (2015).

51. Silva Herran, D., Fujimori, S. \& Kainuma, M. Implications of Japan's long term climate mitigation target and the relevance of uncertain nuclear policy. Clim. Policy 19, 1117-1131 (2019).

52. Clarke, L. E. et al. Technology and US emissions reductions goals: results of the EMF 24 modeling exercise. Energy J. 35, 9-32 (2014).

53. Knopf, B. et al. Beyond 2020-strategies and costs for transforming the European energy system. Clim. Change Econ. 4, 1340001 (2013).

54. O'Neill, B. et al. A new scenario framework for climate change research: the concept of shared socioeconomic pathways. Climatic Change 122, 387-400 (2014).

55. Sugiyama, M. Climate change mitigation and electrification. Energy Policy 44, 464-468 (2012).

56. Fujimori, S., Oshiro, K., Shiraki, H. \& Hasegawa, T. Energy transformation cost for the Japanese mid-century strategy. Nat. Commun. 10, 4737 (2019).

57. The Common Integrated Assessment Model (IAM) Documentation (IAM, 2018); https://www.iamcdocumentation.eu/index.php/IAMC_wiki
58. Riahi, K. et al. The Shared Socioeconomic Pathways and their energy, land use, and greenhouse gas emissions implications: an overview. Glob. Environ. Change Hum. Policy Dimen. 42, 153-168 (2017).

59. Huppmann, D. et al. The MESSAGE ix $_{i x}$ integrated assessment model and the $i x$ modeling platform (ixmp): an open framework for integrated and cross-cutting analysis of energy, climate, the environment, and sustainable development. Environ. Model. Softw. 112, 143-156 (2019).

60. Calvin, K. et al. GCAM v5.1: representing the linkages between energy, water, land, climate, and economic systems. Geosci. Model Dev. 12, 677-698 (2019)

61. Grant, N., Hawkes, A., Napp, T. \& Gambhir, A. The appropriate use of reference scenarios in mitigation analysis. Nat. Clim. Change 10, 605-610 (2020).

62. Kriegler, E. et al. Diagnostic indicators for integrated assessment models of climate policy. Technol. Forecast. Soc. Change 90, 45-61 (2015).

63. Emmerling, J. et al. The WITCH 2016 Model-Documentation and Implementation of the Shared Socioeconomic Pathways (FEEM, 2016).

64. Fujimori, S., Dai, H., Masui, T. \& Matsuoka, Y. Global energy model hindcasting. Energy 114, 293-301 (2016).

65. Calvin, K., Wise, M., Kyle, P., Clarke, L. \& Edmonds, J. A hindcast experiment using the GCAM 3.0 agriculture and land-use module. Clim. Change Econ. 08, 1750005 (2017).

66. Feijoo, F. et al. The future of natural gas infrastructure development in the United states. Appl. Energy 228, 149-166 (2018).

67. Fujimori, S., Kainuma, M. \& Masui, T. Post-2020 Climate Action: Global and Asian Perspective (Springer, 2017).

Publisher's note Springer Nature remains neutral with regard to jurisdictional claims in published maps and institutional affiliations.

(c) Springer Nature Limited 2021 


\section{Methods}

Overview. We carried out scenario analysis for selected Asian countries (China, India, Indonesia, Japan, Korea, Thailand and Vietnam) and implemented eight scenarios for each country. They all have different reduction rates relative to the base year of 2010. Vietnam and Thailand indicated conditional and unconditional target statements in their NDCs. We thus also simulated variations for these conditional statements. In scenario implementations, we considered planned national policies as much as possible. We used AIM/Hub (formerly AIM/CGE) for the proposed scenario design implementation and as the core tool of this study. It is a computable general equilibrium model and has been extensively applied to assessments of Asian national climate policies in past years ${ }^{68-72}$. In the scenario implementations, we used three major GHG gases $\left(\mathrm{CO}_{2}, \mathrm{CH}_{4}\right.$ and $\left.\mathrm{N}_{2} \mathrm{O}\right)$ for emission coverage, considering that the countries have relatively large emissions of non- $\mathrm{CO}_{2}$ gases. The reductions start from $30 \%$ in all countries because we took into account the fact that Japan's baseline emissions have been quite stable over time and thus it may not be meaningful to see the lower reduction levels (such as $10 \%)$. Finally, a regression analysis was conducted on the scenario results. Note that the scenarios in this study excluded climate change impacts, because global emissions scenarios are needed for each national emission scenario to determine such impacts, which is an important factor for national policymakers to consider ${ }^{61,73-75}$

Simulation model and data. AIM/Hub is a 1-yr-step recursive-type dynamic general equilibrium model covering all regions of the world. The AIM/Hub model includes 42 industrial classifications. To assess bioenergy and land-use competition, agricultural sectors are disaggregated ${ }^{76}$. The details of the model structure and mathematical formulae have been described previously 77,78 . Version 2.2 of the AIM/Hub model was used, and the main revisions from the previous version are described below.

Production sectors were assumed to maximize profits using multinested constant elasticity substitution functions and input prices. For energy transformation sectors, to handle energy conversion efficiency appropriately in these sectors, input energy and value added were fixed coefficients of the output. Power generation values from several energy sources were combined with a logit function ${ }^{79}$. This functional form was used to ensure energy balance, as it was not guaranteed by the constant elasticity substitution function. Electricity and bioenergy were produced by multiple sectors (for example, coal-fired, nuclear and solar, agricultural residue, energy crops and sugarcane), which were aggregated by the logit function so that energy production by individual sectors was balanced to match total generation. Household expenditures on each commodity were described with a linear expenditure system function. The parameters adopted in the linear expenditure system function were recursively updated in accordance with income elasticity assumptions. The savings ratio was endogenously determined to balance savings and investment, and capital formation for each good was assigned a fixed coefficient as an exogenous assumption. The Armington assumption was used for trade (using constant elasticity substitution and the constant elasticity of transformation function), and the current account was assumed to be balanced.

In addition to energy-related $\mathrm{CO}_{2}, \mathrm{CO}_{2}$ from other sources, $\mathrm{CH}_{4}, \mathrm{~N}_{2} \mathrm{O}$ and fluorinated gases were treated as GHGs in the model. Energy-related emissions were associated with fossil fuel combustion. Non-energy-related $\mathrm{CO}_{2}$ emissions consisted of changes in land use and industrial processes. Emissions from changes in land use were derived from the change in forest area relative to the previous year multiplied by the carbon stock density, which was differentiated into agro-ecological zones. Non-energy-related emissions other than those associated with changes in land use were assumed to be proportional to the level of each activity (for example, based on output). $\mathrm{CH}_{4}$ emissions arise from a range of sources, mainly rice production, livestock, fossil fuel mining and waste management. $\mathrm{N}_{2} \mathrm{O}$ is emitted as a result of fertilizer application and livestock manure management, as well as by the chemical industry. Fluorinated gases are emitted mainly from refrigerants used in air conditioners and industrial cooling devices. Air pollutant gases (black carbon, $\mathrm{CO}, \mathrm{NH}_{3}$, non-methane volatile organic compounds, $\mathrm{NO}_{x}$, organic carbon and $\mathrm{SO}_{2}$ ) are also associated with fuel combustion and activity levels. Emission factors change over time with the implementation of air pollutant removal technologies and other regulations $s^{80}$

The implementation of mitigation actions in the model was represented by constraints on $\mathrm{CO}_{2}$ emissions. The carbon price was imposed on $\mathrm{CO}_{2}$ as well as other GHG types, such as $\mathrm{CH}_{4}$ and $\mathrm{N}_{2} \mathrm{O}$, arising from every sector. The carbon price increased the price of fossil fuel-based goods when emissions were constrained and promoted energy savings and substitution away from fossil fuels to sources and transport methods with lower GHG emissions. The carbon tax also functioned as an incentive to reduce non-energy-related emissions. Gases other than $\mathrm{CO}_{2}$ were weighted on the basis of their global warming potential and summed as total GHG emissions. Further parameter settings and changes under the future scenarios are documented in Fujimori et al. ${ }^{81}$.

The main revisions from version 2.0, which was used in SSP quantification ${ }^{72}$, to version 2.2 are described in Fujimori et al. ${ }^{82}$, and the most relevant one for this study is the reflection of historical energy data (2005 to 2015). This methodology is the same as model integration with an energy system model, where we exogenously provided the final energy, transport energy share and power energy technological share, while the corresponding parameters in the production function and household consumption were endogenized. Consequently, the autonomous energy efficiency in energy consumption and logit share parameters used to determine the share of power generation by different technologies were calibrated during that period and then used for the future scenarios (for more methodological details, see Fujimori et al. ${ }^{56}$ ). We used the IEA Energy Balances as the historical energy information ${ }^{83}$.

National policies. We adopted current national policies that can be considered relevant for the scenarios as much as possible. The NDCs were all taken into account as emissions constraints for the year 2030. For all countries, population and GDP projections were based on the national perspective until either 2030 or 2035. Rates of SSP2 annual change were extrapolated afterwards. There are some vital energy and climate mitigation-related policies at national levels that were reflected as either model constraints or as reference information to serve as a check that the scenarios are not far from the corresponding national perspectives. For example, in China, the next five-year plan, to be implemented in 2021, is scheduled to be published in late 2020 to early 2021, and thus we decided not to use the latest available five-year plan but have incorporated the best available current energy information. Another example is Thailand, where the power development plan established by the Ministry of Environment ${ }^{84-86}$ was used for model constraints. The full list of national policy information considered in this study is shown in Supplementary Table 3.

Effort sharing. To map the national scenarios with global goals, we used multiple effort sharing schemes shown by van den Berg et al..$^{42}$. For the global scenarios, we adopted the latest global scenarios from the IPCC Special Report on $1.5^{\circ} \mathrm{C}$ database ${ }^{87}$ by taking minimum, median and maximum ranges of IAMs pathways categorized as $1.5^{\circ} \mathrm{C}$ or $1.5^{\circ} \mathrm{C}$-consistent and $2^{\circ} \mathrm{C}$ or $2^{\circ} \mathrm{C}$-consistent for 1.5 and $2{ }^{\circ} \mathrm{C}$ goals, respectively, regardless of the scale of global mean temperature overshoot.

Regression analysis of the scenario indicators. A regression analysis was carried out for the cross-country comparative assessment. The aim of this regression is to derive the general relationships which can be observed in multiple countries between each indicator and reduction levels. The equation applied is shown below.

$$
Y_{r, s}=a X_{r, s}+b_{r}+c+\epsilon_{r, s}
$$

Where:

$Y_{r, s}$ is an individual six indicators (annual mean rate of energy intensity change, annual rate of mean carbon intensity change, share of low-carbon energy sources, electrification rate, carbon price and GDP loss rates) in country $r$ and scenario $s, X_{r, s}$ is the emissions reduction percentage relative to those of 2010.a, $b_{r}$ and $c$ represent estimated parameters and they are the slope of the reduction levels, dummy countries and intercept respectively; $\varepsilon$ is an error term.

\section{Data availability}

Scenario data are accessible online via the ENGAGE Scenario Database at https:// data.ene.iiasa.ac.at/engage/. Data derived from the original scenario database, which are shown as figures but are not in the above database, are available upon reasonable request from the corresponding author. The scenario name mapping table between this paper and the database is shown in Supplementary Table 3. Source data are provided with this paper.

\section{Code availability}

All code used for data analysis and creating the figures is available via GitHub at https://doi.org/10.5281/zenodo.4677638 (ref. ${ }^{88}$ ).

\section{References}

68. Fujimori, S., Kainuma, M., Masui, T., Hasegawa, T. \& Dai, H. The effectiveness of energy service demand reduction: a scenario analysis of global climate change mitigation. Energy Policy 75, 379-391 (2014).

69. Fujimori, S., Masui, T. \& Matsuoka, Y. Gains from emission trading under multiple stabilization targets and technological constraints. Energy Econ. 48, 306-315 (2015)

70. Thepkhun, P., Limmeechokchai, B., Fujimori, S., Masui, T. \& Shrestha, R. M. Thailand's low-carbon scenario 2050: the AIM/CGE analyses of $\mathrm{CO}_{2}$ mitigation measures. Energy Policy 62, 561-572 (2013).

71. Hasegawa, T. et al. Consequence of climate mitigation on the risk of hunger. Environ. Sci. Technol. 49, 7245-7253 (2015).

72. Fujimori, S. et al. SSP3: AIM implementation of Shared Socioeconomic Pathways. Glob. Environ. Change Hum. Policy Dimen. 42, 268-283 (2017).

73. Burke, M., Hsiang, S. M. \& Miguel, E. Global non-linear effect of temperature on economic production. Nature 527, 235-239 (2015).

74. Hsiang, S. et al. Estimating economic damage from climate change in the United States. Science 356, 1362-1369 (2017) 
75. Glanemann, N., Willner, S. N. \& Levermann, A. Paris Climate Agreement passes the cost-benefit test. Nat. Commun. 11, 110 (2020).

76. Fujimori, S., Hasegawa, T., Masui, T. \& Takahashi, K. Land use representation in a global CGE model for long-term simulation: CET vs. logit functions. Food Secur. 6, 685-699 (2014).

77. Fujimori, S., Masui, T. \& Matsuoka, Y. AIM/CGE [Basic] Manual Report No. 2012-01 (Center for Social and Environmental Systems Research, National Institute of Environmental Studies, 2012).

78. Fujimori, S., Masui, T. \& Matsuoka, Y. in Post-2020 Climate Action: Global and Asian Perspective (eds Fujimori, S. et al.) 201-303 (Springer, 2017).

79. Sands, R. D. Dynamics of carbon abatement in the second generation model. Energy Econ. 26, 721-738 (2004).

80. Rao, S. et al. Future air pollution in the Shared Socio-economic Pathways. Glob. Environ. Change Hum. Policy Dimen. 42, 346-358 (2017).

81. Fujimori, S., Hasegawa, T. \& Masui, T. in Post-2020 Climate Action: Global and Asian Perspective (eds Fujimori, S. et al.) 305-328 (Springer, 2017)

82. Fujimori, S. et al. Measuring the sustainable development implications of climate change mitigation. Environ. Res. Lett. 15, 085004 (2020).

83. World Energy Balances (OECD/IEA, 2018).

84. Thailand Power Development Plan 2015-2036 (PDP2015) (Energy Policy and Planning Office, Ministry of Energy, 2015).

85. Guidelines for Upgrading Thai Electricity Generation Plans (Ministry of Energy, 2018).

86. Power Development Plan 2561-2580 (PDP2018) (Ministry of Energy, 2019).

87. Huppmann, D. et al. IAMC $1.5^{\circ} \mathrm{C}$ Scenario Explorer and Data Hosted by IIASA (IAMC \& IIASA, 2018)

88. Fuijimori, S. Code for A framework for national scenarios with varying emission reductions. Github https://github.com/shinichirofujimoriKU/ AsianMCSAnalysis (2021).

\section{Acknowledgements}

S.F., K.O., D.S.H. and T.M. are supported by the Environment Research and Technology Development Fund (grant numbers JPMEERF20192008 and JPMEERF20211001) of the Environmental Restoration and Conservation Agency of Japan. S.F. and K.O. are supported by JSPS KAKENHI (19H02273) and the Sumitomo Foundation. S.F., K.O., V.K., D.v.V., K.R., B.L. and T.T.T received funding from the European Union's Horizon 2020 research and innovation programme under grant agreement number 821471 (ENGAGE). C.P. was supported by the International Research \& Development Program of the National Research Foundation of Korea (NRF) funded by the Ministry of Science and ICT (grant number NRF2019K1A3A1A7811297311).

\section{Author contributions}

S.F., V.K., D.v.V., M.S. and K.O. designed the research. S.F. and Y.O. carried out the analysis of the modelling results and created the figures. S.F. wrote the draft of the paper. S.F., K.O., O.N. and D.S.H. set up the model. P.C., S.M., C.P., D.S.H., T.T.T. and S.Z. ran the model simulations and P.C., S.M., C.P., D.S.H., T.T.T., P.V.H.N. and S.Z. provided national policy information. All authors contributed to writing the manuscript.

\section{Competing interests}

The authors declare no competing interests.

\section{Additional information}

Supplementary information The online version contains supplementary material available at https://doi.org/10.1038/s41558-021-01048-z.

\section{Correspondence should be addressed to S.F.}

Peer review information Nature Climate Change thanks Ritu Mathur, Roberto Schaeffer and the other, anonymous, reviewer(s) for their contribution to the peer review of this work.

Reprints and permissions information is available at www.nature.com/reprints. 